\title{
Ceria Nanoparticles Ameliorate Renal Fibrosis by Modulating the Balance Between Oxidative Phosphorylation and Aerobic Glycolysis
}

\section{Wang mengling}

Fudan University School of Pharmacy

\section{Zeng feng}

Guangzhou University of Chinese Medicine

\section{Ning fengling}

Fudan University School of Pharmacy

\section{Wang yinhang}

Fudan University School of Pharmacy

He jiaqi

Fudan University School of Pharmacy

\section{Zhou shilin}

Fudan University School of Pharmacy

\section{Li cong}

Fudan University School of Pharmacy

\section{Zhang dongliang}

fudan daxue fushu zhongshan yiyuan minhang fenyuan: Fudan University Minhang Hospital

Hu ping

fudan daxue fushu minhang yiyuan: Fudan University Minhang Hospital

\section{Xin hong}

Fudan University School of Pharmacy

\section{Xu xudong}

fudan daxue fushu minhang yiyuan: Fudan University Minhang Hospital

Zhang xuemei ( $\nabla$ xuemzhang@shmu.edu.cn)

Fudan University School of Pharmacy

\section{Research}

Keywords: Ceria nanoparticles, Metabolic reprogramming, Oxidative phosphorylation, Aerobic glycolysis, Renal fibrosis

Posted Date: May 29th, 2021 
DOl: https://doi.org/10.21203/rs.3.rs-544845/v1

License: (c) (1) This work is licensed under a Creative Commons Attribution 4.0 International License. Read Full License 


\section{Abstract}

Background and aims: Renal fibrosis is the common outcome in all progressive forms of chronic kidney disease. Unfortunately, the pathogenesis of the renal fibrosis remains largely unexplored, among which metabolic reprogramming plays an extremely crucial role in the evolution of renal fibrosis. Ceria nanoparticles have strong ROS scavenging and anti-inflammatory activity. The present study aims to determine whether CeNPPEG has therapeutic value for renal fibrosis.

Methods: Ceria nanoparticles and Ceria nanoparticles-PEG were synthesized according to previously reported procedures with slight modifications. For the in vivo studies, unilateral ureteral obstructive induced kidney fibrosis was chosen. Through cellular studies, TGF- $\beta 1$-induced epithelial-to-mesenchymal transition in HK-2 cells of enhanced glycolysis was initially utilized to report the associations among Ceria nanoparticles-PEG, oxidative phosphorylation, glycolysis and renal fibrosis.

Results: In this study, Ceria nanoparticles were developed and the Ceria nanoparticles-PEG treatment significantly ameliorated renal fibrosis in unilateral ureteral obstructive mice as well as protected HK-2 cells from transforming growth factor beta 1 -induced epithelial-to-mesenchymal transition process. Furthermore, we found that Ceria nanoparticles-PEG suppressed hexokinase 1and hexokinase 2 to block the dysregulated metabolic status, in which damaged epithelial cells take glycolytic metabolism as priority to oxidative phosphorylation, exerting the protective function in the progress of renal fibrosis.

Conclusions: The application of Ceria nanoparticles-PEG in this study is attempted to provide another therapeutic option on renal fibrosis.

\section{Full-text}

Due to technical limitations, full-text HTML conversion of this manuscript could not be completed. However, the manuscript can be downloaded and accessed as a PDF.

\section{Figures}


A

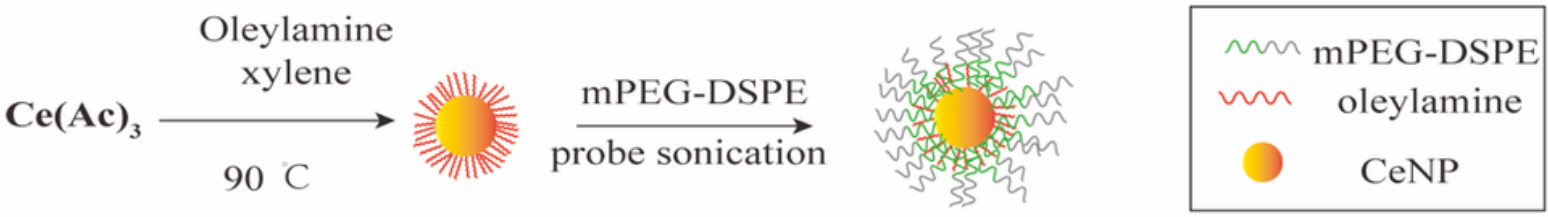

B

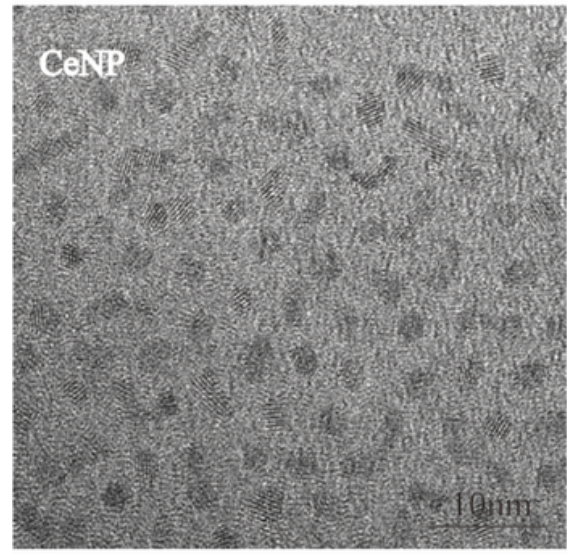

D

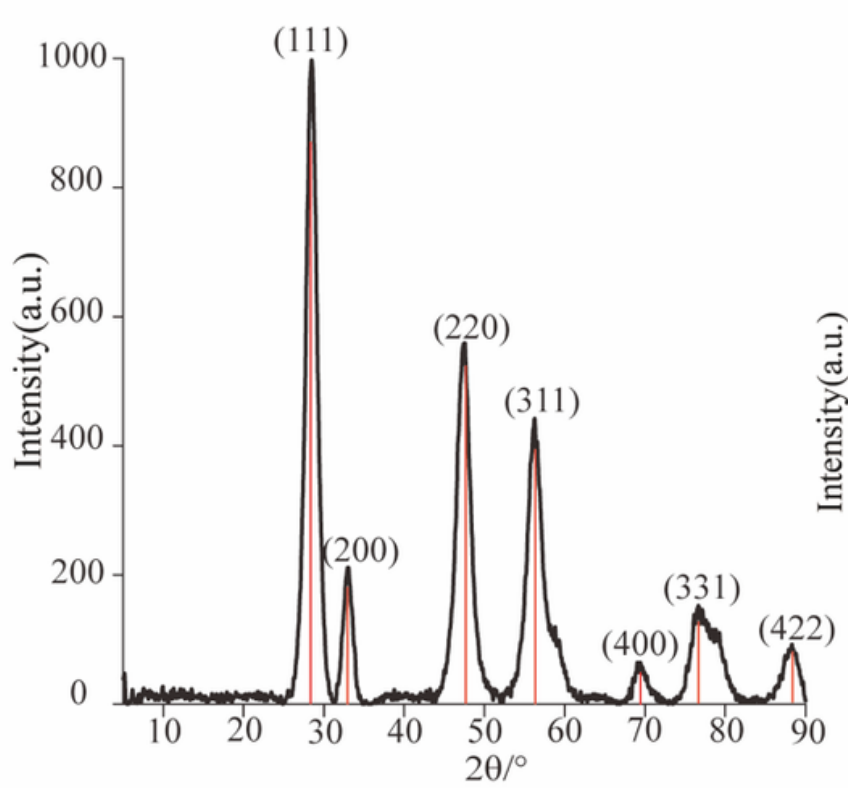

CeNP-PEG

C

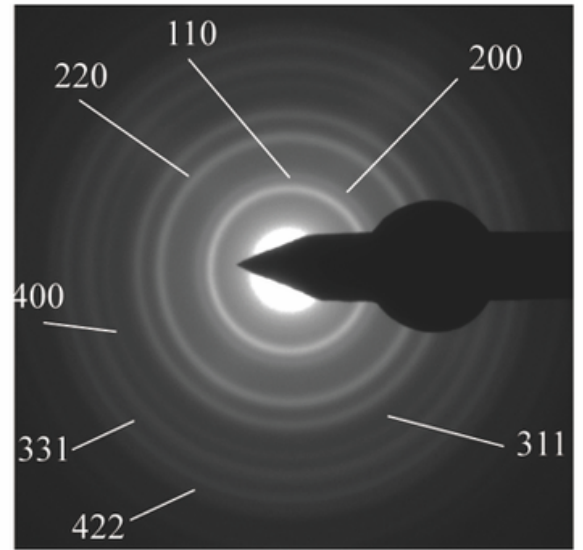

$\mathbf{E}$

- Experimental

— Peak Sum

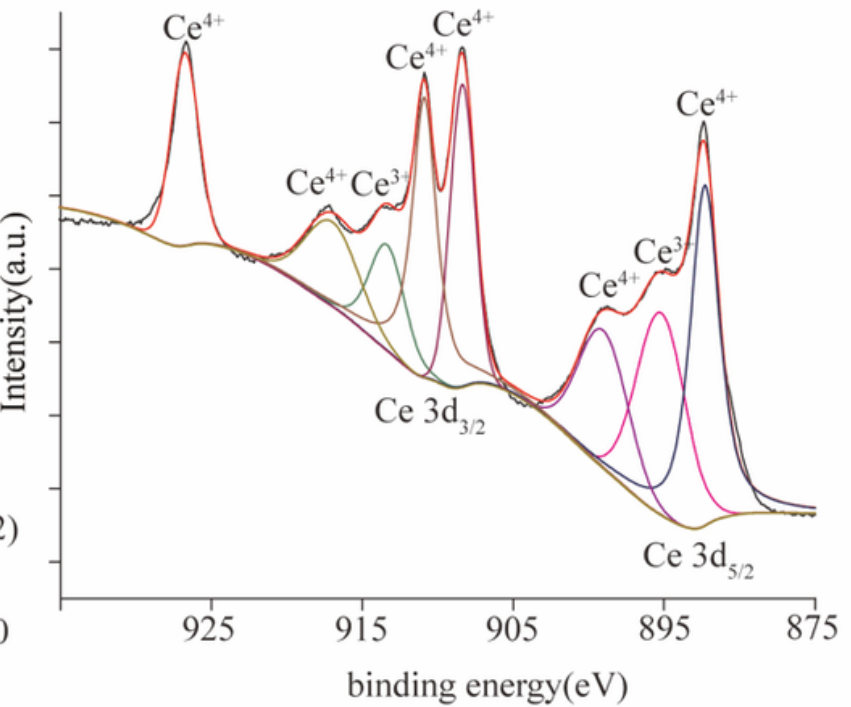

\section{Figure 1}

Synthesis and characterization of CeNP-PEG. (A) Synthetic procedure of CeNP and CeNP-PEG. (B) Representative high resolution TEM images of CeNP and CeNP-PEG. The SAED (C) and XRD (D) images reveal the cubic fluorite structure of the CeNP. (E) The XPS spectra of ceria nanoparticles reveal the valence state and the corresponding binding energy peaks of 885.2 and $903.4 \mathrm{eV}$ for $\mathrm{Ce} 3+$ and 882.3 , 889.0, 898.4, 900.9, 907.0 and $916.8 \mathrm{eV}$ for Ce4+. a.u.: arbitrary units 

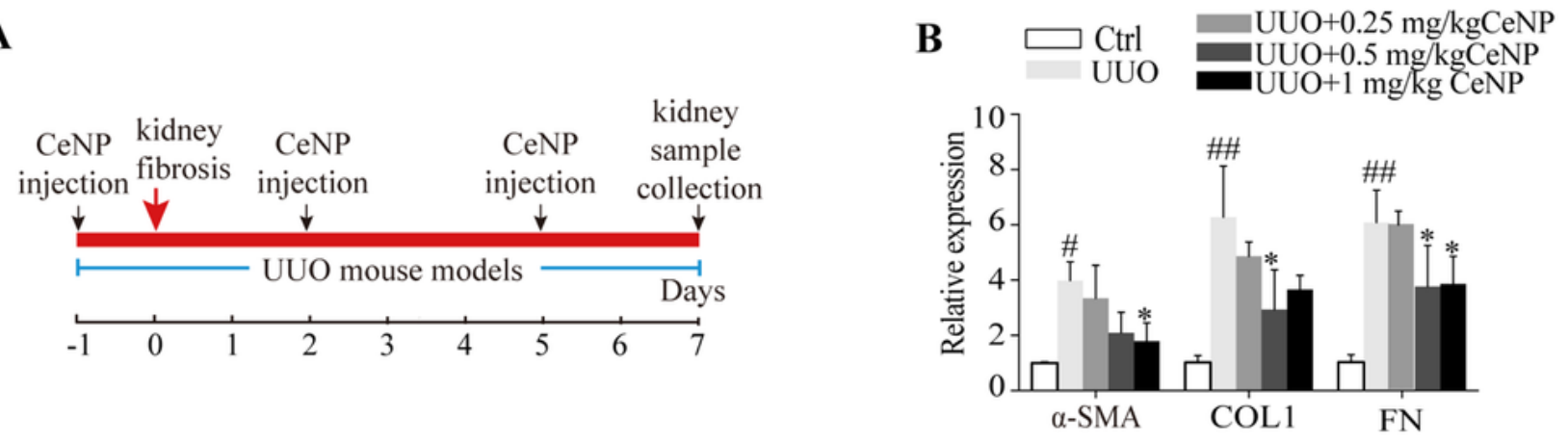

C

$\begin{array}{rcccccccccc}\text { UUO } & - & - & + & + & + & + & + & + & + & + \\ \mathrm{CeNP}(\mathrm{mg} / \mathrm{kg}) & - & - & - & - & 0.25 & 0.25 & 0.5 & 0.5 & 1.0 & 1.0\end{array}$

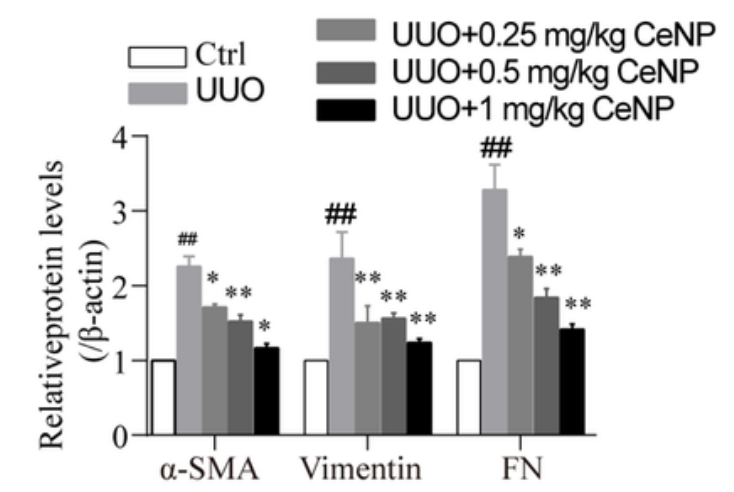

\section{D}

UUO

$\mathrm{CeNP}(\mathrm{mg} / \mathrm{kg})$
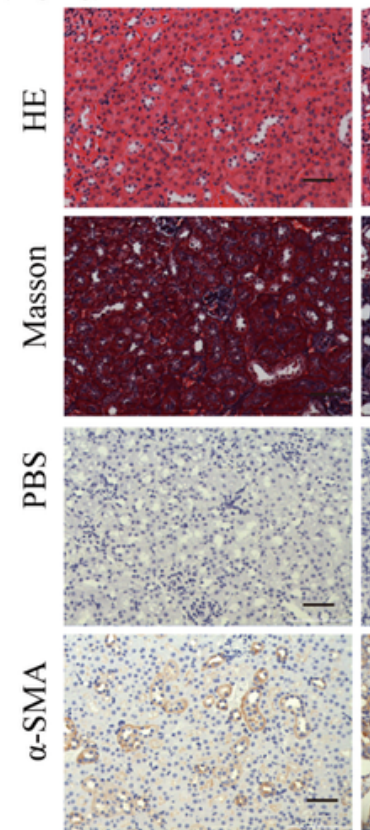

ตับที1

$270 \mathrm{KDa}$

$57 \mathrm{KDa}$

$42 \mathrm{KDa}$

$42 \mathrm{KDa}$

0.25
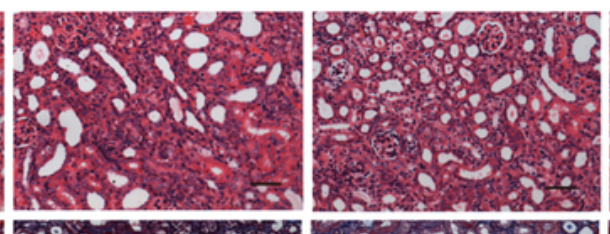

0.5

1.0

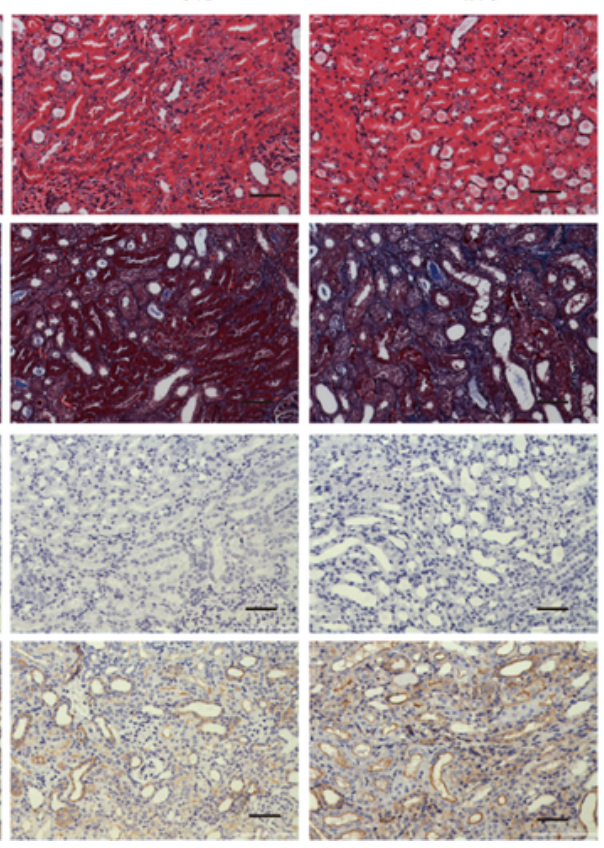

Figure 2

CeNP-PEG ameliorated renal fibrosis in UUO mice (A) Schematic graph of experimental design. (B) The expression of $\mathfrak{a}-\mathrm{SMA}, \mathrm{COL} 1$ and FN was evaluated by RT-PCR analysis. $\beta$-actin was used as the control (\#P<0.05 and \#\#P<0.01 for UUO versus control, and ${ }^{*} \mathrm{P}<0.05$ and ${ }^{* *} \mathrm{P}<0.01$ for UUO versus UUO+CeNPPEG; $n=3$ for each group). (C) The expression of $\mathfrak{a}-S M A$, Vimentin and FN was evaluated by western blotting analysis. $\beta$-actin was used as the loading control $(\# \mathrm{P}<0.05$ and \#\#P<0.01 for UUO versus control, 
and $* P<0.05$ and $* * P<0.01$ for 684 UUO versus UUO+CeNP; $n=3$ for each group). (D) Renal injury was evaluated by hematoxylin and eosin staining. The renal fibrosis was evaluated by Masson, trichrome and immunohistochemical analysis for the a-SMA (c) expression. PBS was used as the negative control.

A

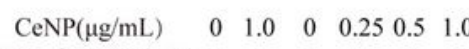
TGF- $\beta 1(10 \mathrm{ng} / \mathrm{mL})-\quad++++$

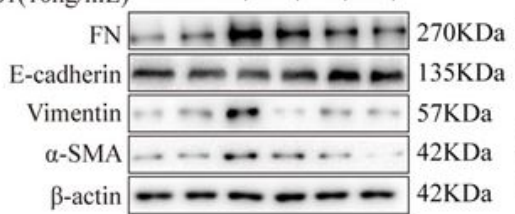

B

$\mathrm{CeNP}(\mu \mathrm{g} / \mathrm{mL}) \quad 0 \quad 1.0 \quad 0 \quad 0.250 .5 \quad 1.0$ TGF- $\beta 1(10 \mathrm{ng} / \mathrm{mL})$ -
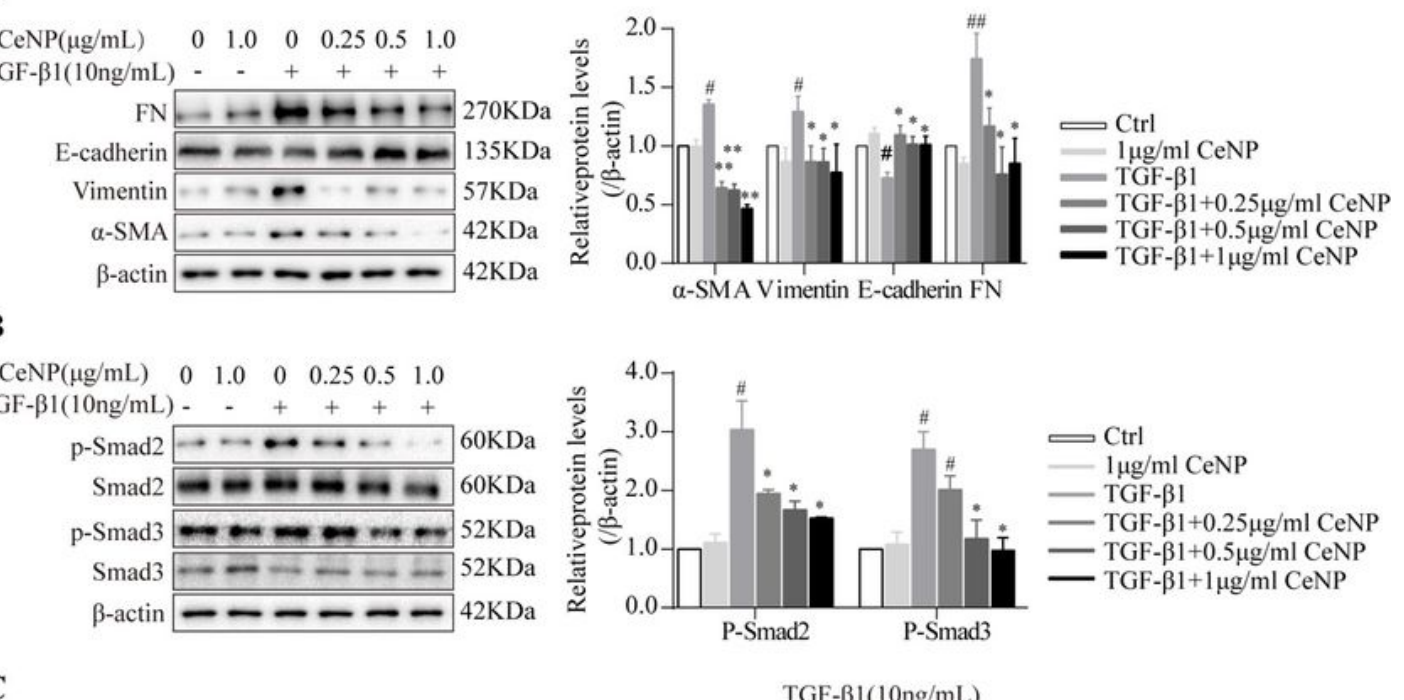

C
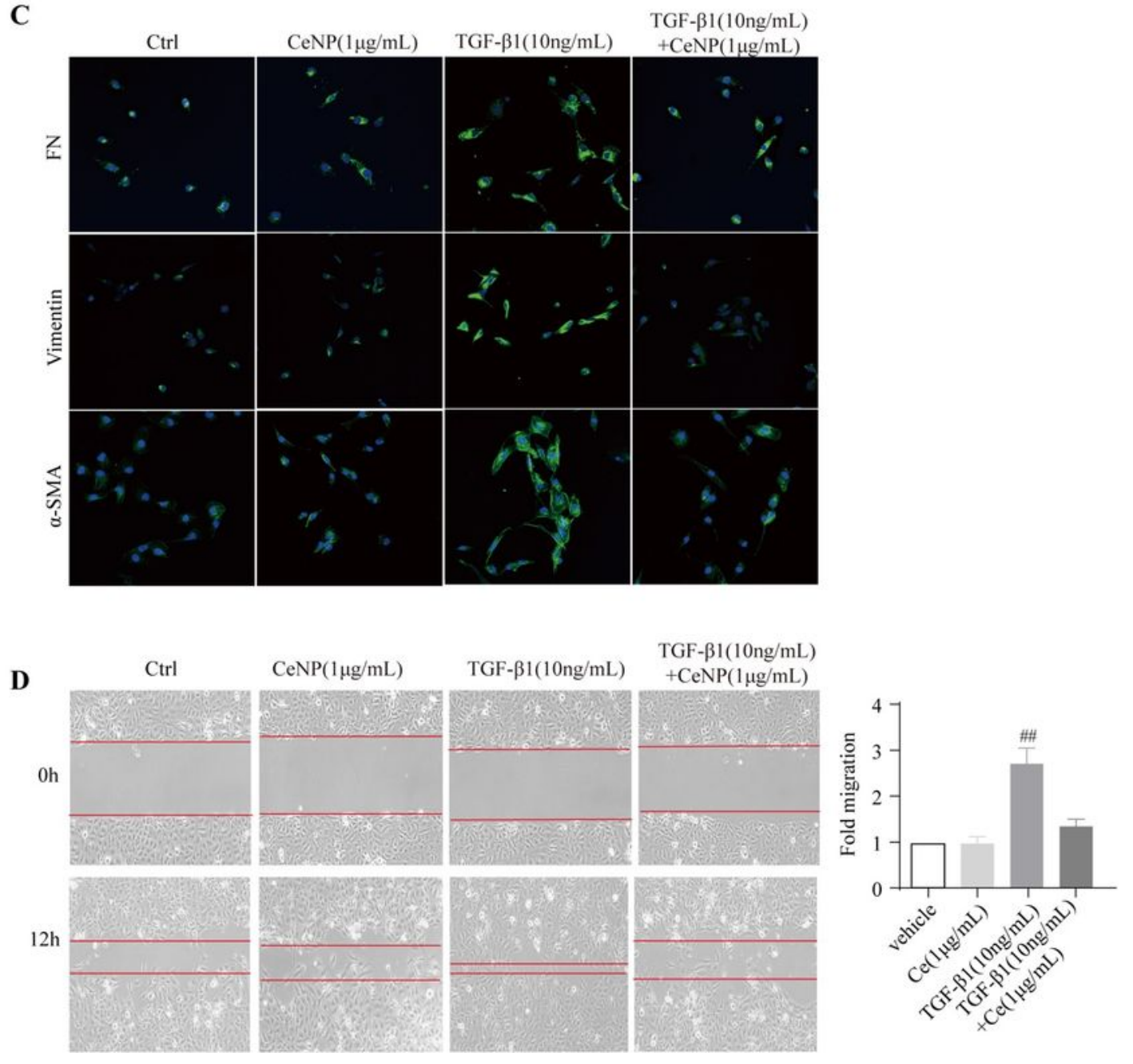

Figure 3

CeNP-PEG protected HK-2 cell from TGF- $\beta 1$ induced EMT process. (A) The expression of $\mathfrak{a}$-SMA, Vimentin, FN and E-cadherin was evaluated by western blotting analysis. $\beta$-actin was used as the loading 
control. (B) The expression of phosphorylated and total Smad2 and Smad3 was evaluated by western blotting analysis. $\beta$-actin was used as the loading control 693 (\#P<0.05 and \#\#P<0.01 for TGF- $\beta 1$ versus control, and ${ }^{*} \mathrm{P}<0.05$ and ${ }^{*} \mathrm{P}<0.01$ for TGF- $\beta 1$ versus TGF- $\beta 1+C e N P ; n=3$ for each group). (C)The immunohistochemical analysis was performed on $\mathfrak{a}$-SMA, Vimentin and FN in HK-2 cells. Original magnification: $\times 200$. All data were presented as mean \pm SD.(D) Scratch assays was performed on HK-2 cell treated with TGF- $\beta 1$ and/or CeNP-PEG and wound closure was quantified. Red lines indicate leading edge after $12 h$ ( $n=3$ for each group).

A
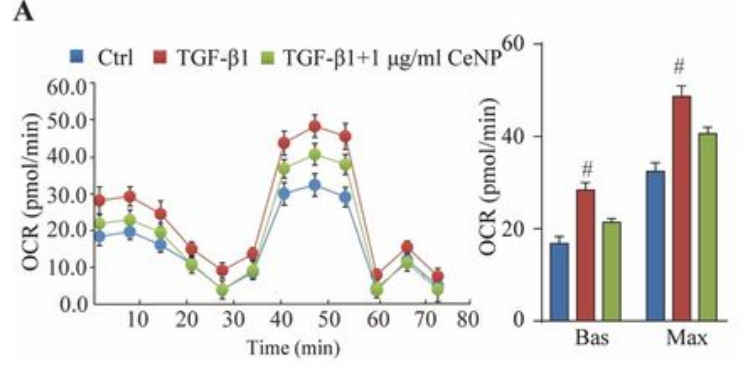

B
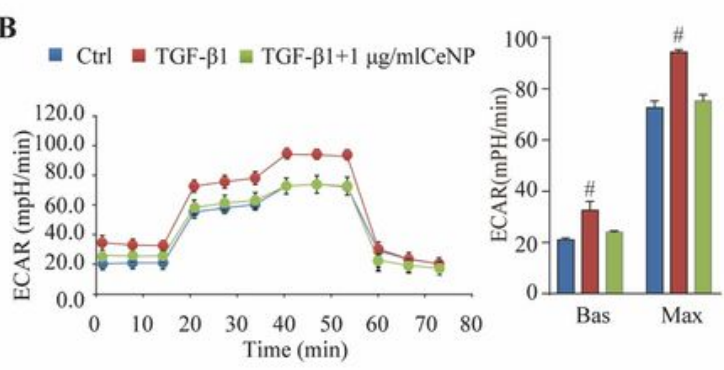

C

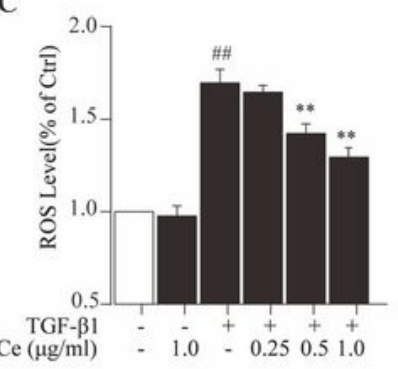

D

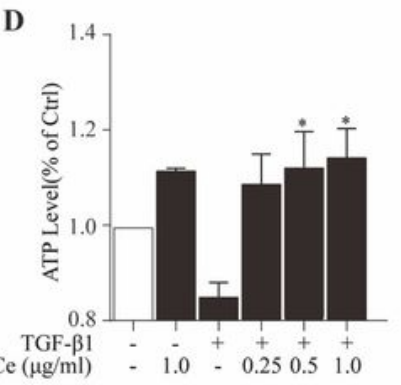

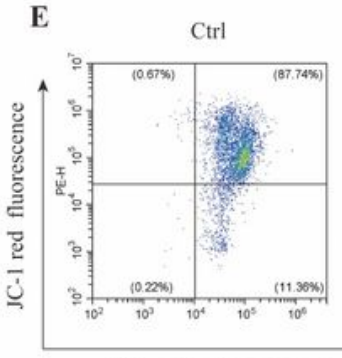
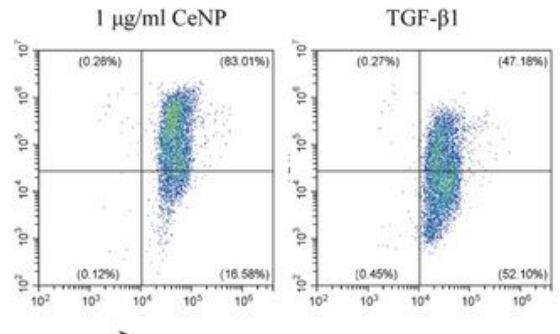

TGF- $\beta 1+1 \mu \mathrm{g} / \mathrm{ml} \mathrm{CeNP}$

JC-1 green fluorescence

F

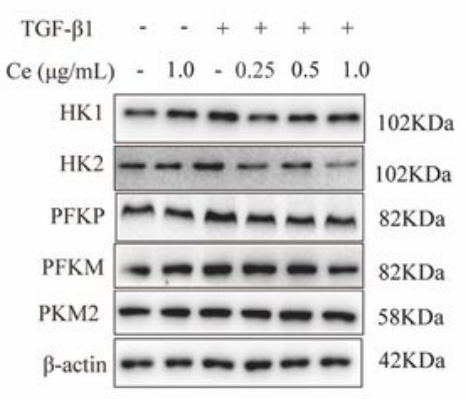

G
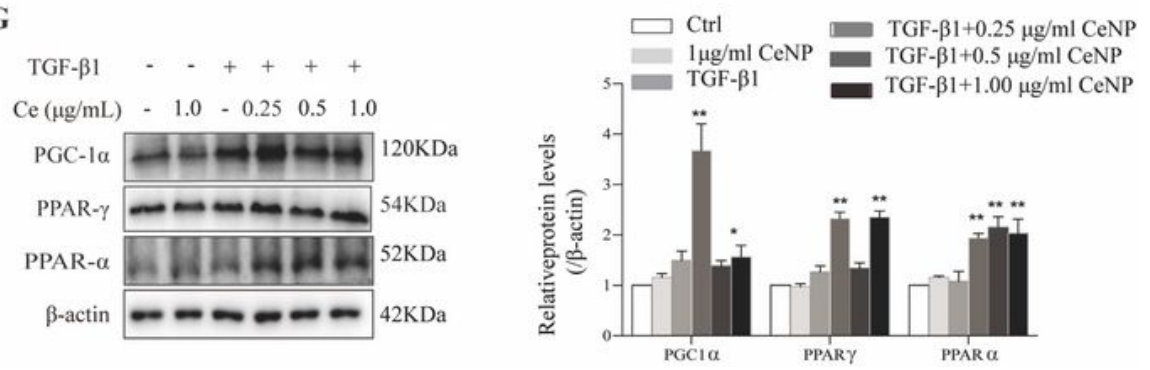


\section{Figure 4}

CeNP-PEG blocked aerobic glycolysis 701 and enhanced OXPHOS, contributing to EMT suppression in vitro (A) OCR measurements of the mitochondrial stress test and (B) ECAR measurements of the glycolysis stress test were performed in HK-2 cells treated with or without TGF- $\beta 1$, and/or CeNP-PEG. The Basic and maximum capacity of OCR and ECAR were quantified. (C) The ROS levels of cells treated with TGF- $\beta 1$ and/or CeNP-PEG was determined using the fluorescence spectrophotometry. (D)The ATP content of cells treated with TGF- $\beta 1$ and/or CeNP PEG was determined using the ATP Bioluminescence Assay Kit. (E) Mitochondrial membrane potential of cells treated with TGF- $\beta 1$ and/or CeNP-PEG was measured by flow cytometry. $(F, G)$ The expression of HK1/2, PFKP, PFKM ,PKM2, PGC1a,PPARa and PPARystimulated by TGF- $\beta 1$ and/or CeNP-PEG or 2-DG was evaluated by western blotting analysis. $\beta$-actin was used as the loading control, all data were presented as mean \pm SD $(\# P<0.05$ and \#\#P<0.01 for TGF- $\beta 1$ versus control, and $* P<0.05$ and $* * P<0.01$ for TGF- $\beta 1+C e N P-P E G$ and TGF- $\beta 1+2-D G ; n=3$ for each group). 
A

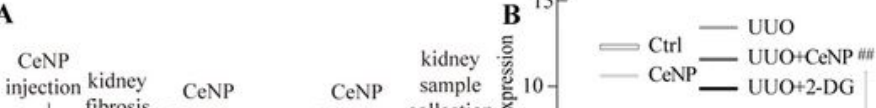

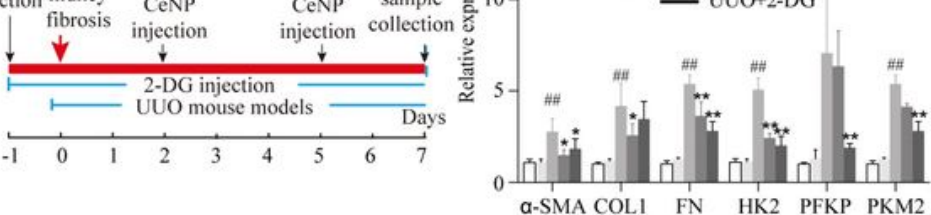

C

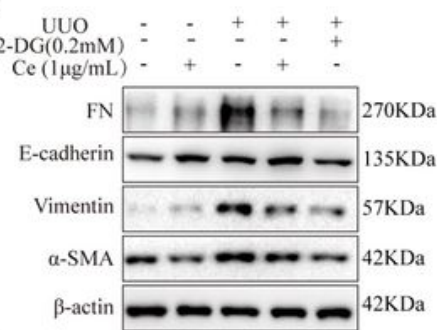

D
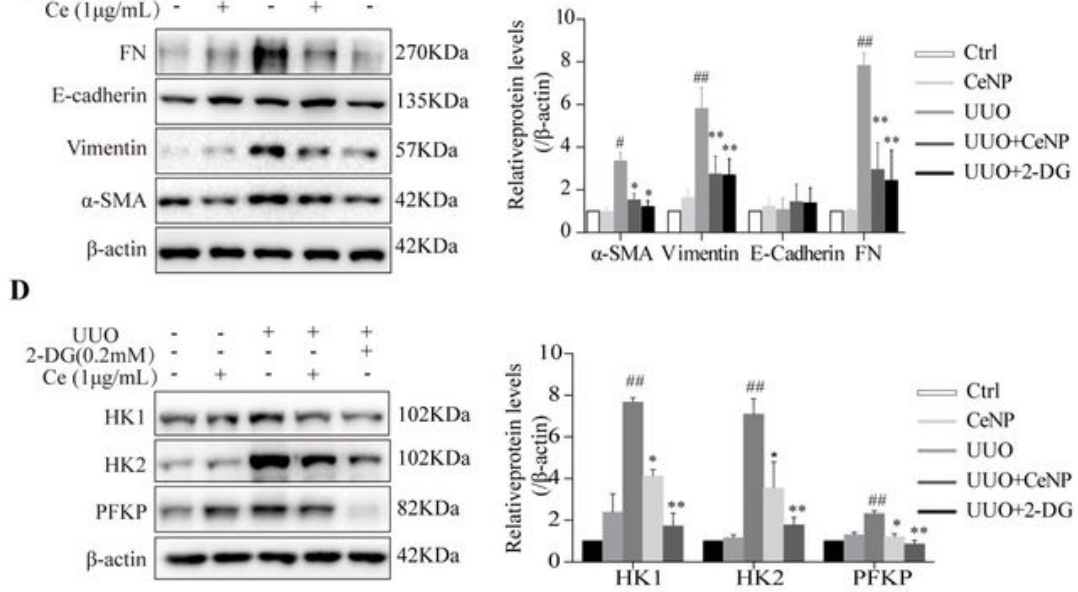

E
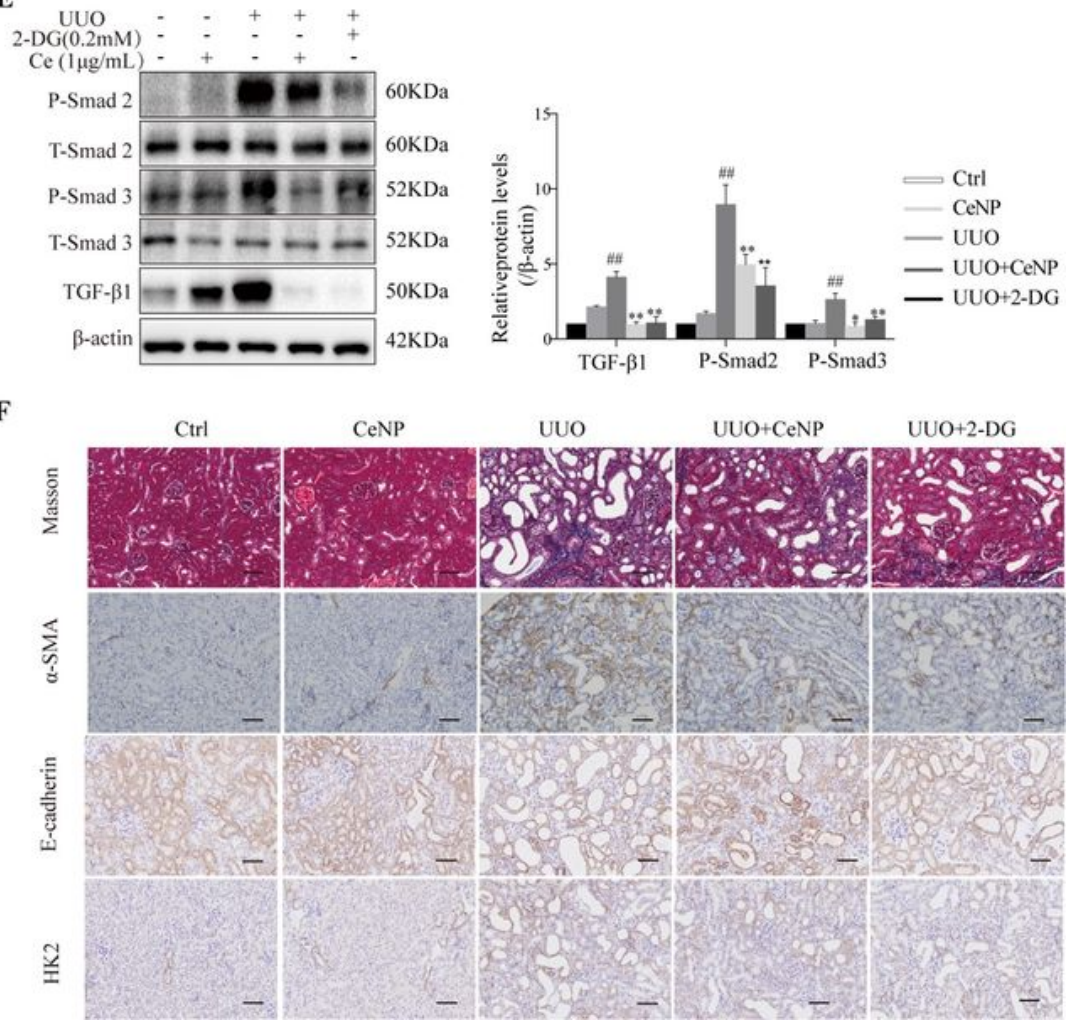

Figure 5

CeNP-PEG inhibited aerobic 716 glycolysis in UUO mice.(A) The schematic graph of the experimental design. (B) The expression of $\mathfrak{a}-\mathrm{SMA}, \mathrm{COL} 1, \mathrm{FN}, \mathrm{HK} 2, \mathrm{PFKP}$ and PKM2 was evaluated by RT-PCR analysis. $\beta$-actin was used as the control (\#P<0.05 and \#\#P<0.01 for UUO versus control, and $* P<0.05$ and ${ }^{*} \mathrm{P}<0.01$ for UUO versus UUO+CeNP-PEG; $n=3$ for each group). (C) The expression of $\mathfrak{a}$-SMA, E-cadherin, Vimentin and FN was evaluated by western blotting analysis. $\beta$-actin was used as the control. (D) The 
expression of HK1,HK2 and PFKP was evaluated by western blotting analysis. $\beta$-actin was used as the control. (E) The expression of phosphorylated and total Smad2 and Smad3 was evaluated by western blotting analysis. $\beta$-actin was used as the loading control. (\#P<0.05 and \#\#P<0.01 for UUO versus control, and ${ }^{*} \mathrm{P}<0.05$ and $* * \mathrm{P}<0.01$ for UUO versus UUO+CeNP-PEG; $\mathrm{n}=3$ for each group). ( $F$ ) kidney fibrosis was evaluated by Masson trichrome, and immunohistochemical analysis was performed for the a-SMA, ECadherin and HK2 expression.

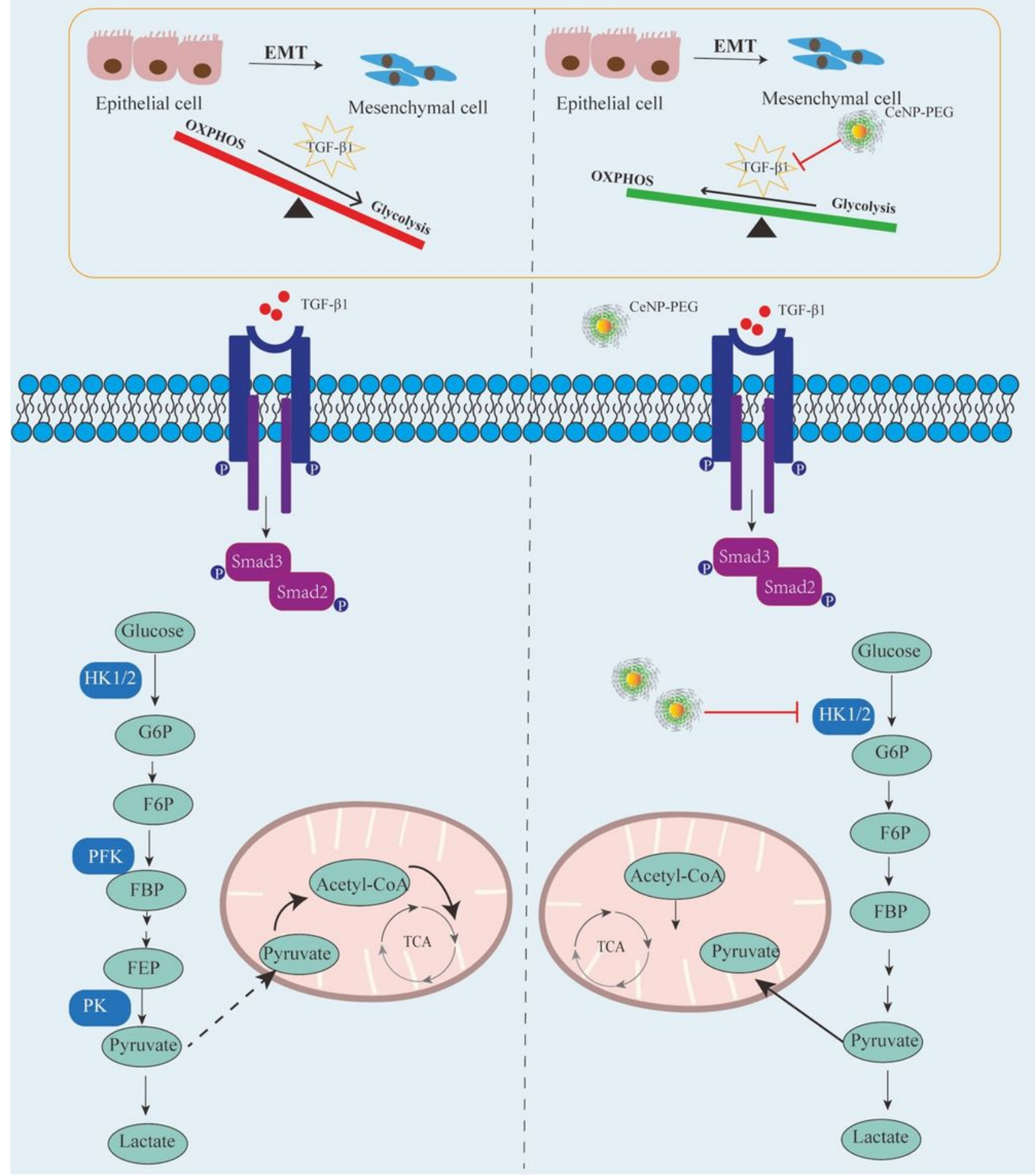

Figure 6 
CeNP-PEG reverses renal fibrosis by blocking the dysregulated metabolic status, in which oxidative phosphorylation switches to aerobic glycolytic program. In normal conditions, renal epithelial cells prefer oxidative phosphorylation for energy supply. During EMT process, TGF- $\beta 1$ induces metabolic reprogramming in renal epithelial cells and takes glycolytic metabolism as priority to OXPHOS. While, CeNP-PEG can block it through the suppression of HK1 and HK2 during EMT process, exerting the protective function in the progress of kidney fibrosis.

\section{Supplementary Files}

This is a list of supplementary files associated with this preprint. Click to download.

- Graphicalabstract.docx

- sTable1.docx 